\title{
The Effect of Demand Response in the Minimum Available Reserve of Energy Management
}

\author{
João Soares, Student Member, Nuno Borges, Ali Fotouhi, Zita Vale, Senior Member \\ GECAD - Knowledge Engineering and Decision-Support Research Centre \\ Polytechnic of Porto (ISEP/IPP) \\ Rua Dr. António Almeida, 431, 4200-072, Porto, Portugal \\ \{joaps, ndsbs, mafgh, zav\}@isep.ipp.pt
}

\begin{abstract}
This paper presents a multi-objective energy scheduling for the daily operation of a Smart Grid (SG) considering maximization of the minimum available reserve in addition to the cost minimization, to take into account the reliability requirements of critical and vulnerable loads. A Virtual Power Player (VPP) manages the day-ahead energy resource scheduling in the smart grid, considering Distributed Generation (DG) and Vehicle-To-Grid (V2G), while maintaining a highly reliable power for the critical loads. This work considers high penetration of critical loads, e.g. industrial processes that require high power quality, high reliability and few interruptions. A mathematical formulation is described and a deterministic technique based on Mixed-Integer Linear Programming (MILP) is used to solve the multi-objective problem. The effect of some customers with DR in this context is analyzed to assess the benefits in the energy scheduling problem. A case study using a 180-bus Portuguese distribution network with 90 load points, several DG units and a large fleet of Electric Vehicles (EVs) with V2G is used to illustrate the performance of the proposed method.
\end{abstract}

Index Terms - Electric Vehicles, Multi-objective optimization, Pareto front, Mixed-Integer Linear Programming, Smart Grid

\section{INTRODUCTION}

The idea behind Smart Grids (SG) is to supply electricity efficiently, reliable, and securely from the generation to the customers [1]-[3]. The integration and proliferation small independent Distributed Energy Resources (DER), such as fuel cells, micro-turbines, combined heat and power units, photovoltaic systems, small wind turbines, biomass, Energy Storage Systems (ESS), e.g. storage batteries, and Electric Vehicles (EVs) is enabled in the SG environment [4], [5]. However, the increasing penetration of DERs in SGs entail important technical and economic factors that need to be considered. On such example is the variability of distributed renewable energy sources (wind and PV), the high maintenance costs and the participation of small producers in the wholesale market are the challenges that must be overcome to take the advantage of the use of DER [6]. The DER's aggregation can provide technical and commercial benefits. By mixing different generation technologies, it is possible to attain

The present work was done and funded in the scope of the following projects: EUREKA - ITEA2 Project SEAS with project number 12004; UID/EEA/00760/2013, and SFRH/BD/87809/2012 funded by FEDER Funds through COMPETE program and by National Funds through FCT. Authors appreciate the network data supplied by EDP Distribuição, S.A. The original network was simplified to suit the objective of the proposed contribution. higher profits and therefore overcome the serious disadvantages of some technologies [7].

Demand Response (DR) programs are being increasingly considered to ensure more reliability and quality of supply to its consumers [8]. DER and DR are not capable of participating in the wholesale market due to their small capacities. Without an aggregator entity such as Virtual Power Player (VPP), which are able to join these small individual units, DER potential is not fully seized. VPPs can operate in many different ways regarding the resources and the network they manage. In this work it is assumed that the VPP manages a physical part of a distribution network or a MicroGrid (MG).

Several multi-objective models have been proposed in the literature to determine the optimal resource scheduling for the day-ahead horizon [9]-[14]. In [9], a multi-objective optimization approach for a microgrid (MG) system that can buy or sell energy with the main grid is proposed. The multiobjective problem takes into account the energy cost and the thermal comfort of the occupants of the MG. In most of the multi-objective models proposed in the literature regarding ERM, emissions minimization is considered in the problem [15], [10]-[14]. The multi-objective formulation developed in [12] does not consider EVs, DR and ESS integrated in the MG model. Moreover, the proposed model in [14], does not consider the presence of EVs neither any type of DR. The authors agree that emissions concern will become less important in MG context due to the increase of renewable based DERs.

For that reason, this work concentrates on another aspect of the MG management, namely the reliability concern on the ERM model through a multi-objective formulation. A deterministic technique, Mixed-Integer Linear Programming (MILP) is used to solve the multi-objective problem. Pareto solutions of the multi-objective model Pareto weighted sum approach, which provide referential schedules for the VPP, to select the most suitable decision, a tradeoff decision between the minimum operation cost and the available reserve during each period of the next day. The idea is minimize the need to buy energy from the day-ahead market or from the external entities at high prices while achieving acceptable level of minimum reserve. That way, we also create two different 
scenarios, one with DR and other without DR, to assess the influence that the use of DR has in the final results. The main contribution of this conference paper is to assess the effect of DR in energy scheduling problem considering minimization of operation costs and maximization of minimum available reserve.

The methodology is evaluated in a realistic case study of a $30 \mathrm{kV}$ distribution network system in Portugal adapted from [16] with one substation, 180 buses, 90 load points and a fleet of 1000 EVs with V2G. This paper is organized as follows: Section II introduces the main features of the proposed model and the problem formulation. Section III describes the case study. Section IV illustrates the results and provides the discussion of the obtained results. Finally, Section V brings the most relevant conclusions of the work.

\section{MATHEMATICAL MODEL}

This section, presents the mathematical model of the multiobjective optimization for the safe operation of a smart grid to help the decision-making process of a VPP. It is assumed that the VPP is geographically in a micro-grid or a part of distribution network but the responsibility of the network conditions (e.g. power flow) is not. In addition to the objective function and constraints, the optimization method is discussed in this section.

\section{A. Energy resource management problem}

The ERM problem in SGs allows the VPPs to obtain the resources that should be dispatched in the next day $(D+1)$ and helps them to decide how much energy should be bought in day-ahead market or from external suppliers. In the ERM model, the load demand is satisfied by the internal DG units and the external suppliers (retailers and electricity pool). The DR programs, ESS and EVs are considered as additional DER. The aggregator controls the fleet's charging process, based on the technical constraints. The fleet can also be used to deliver energy to the grid using the V2G capability. Other inputs of this decision-making framework are the network characteristics, including bus and lines data, the loads and the generators' technical constraints. Moreover, the optimization framework considers the possibility of non-supplied demand (NSD) and generation curtailment power (GCP) penalties related to excess of generation from renewables. The decision variables of the ERM model are the scheduling of the energy resources, such as the output of the dispatchable DG units, the charging schedule and the discharging process of EVs and ESS, and the DR use.

Critical loads and reliability-seeking customers that are susceptible to risk of failures or blackouts are willing to pay the VPP for the higher system costs associated with the higher minimum reserve in the system. Higher reliability is desirable for specific and critical loads, mainly the industrial loads, such as metallurgy and automotive, health centres, hospitals and life safety loads. For example, the life safety loads need to operate when there is an emergency and should operate as long as possible. According to [17], relying on normal system cannot ensure the continuity of operation for those systems. The formulation of the energy resource scheduling problem described in this paper helps the VPPs to find a set of optimal solutions that represent a trade-off between the operation costs and the minimum reserve.

\section{B. Model assumptions}

The proposed model is based on the following assumptions:

- The SG is equipped with the information and communication technologies (ICT), that enables the VPP to monitor and actively control the network;

- The proposed model is adaptable to different scheduling intervals. Nevertheless, in the case study the day-ahead ERM is done for 24 hours;

- The model relies on several inputs, namely high accuracy forecasts. The models of demand forecast are well developed in the literature [14], [18];

- The EVs are not considered in the reserve formulation due to their random behavior.

- The income is assumed to be fixed in the ERM model and only the operation costs are considered;

\section{Objective function}

The two conflicting objectives of the VPP in day actual day $(D)$ are minimizing the total operation cost of the system for the $D+1$ (1) and maximizing the minimum system reserve (2). These objectives are conflicting, because higher minimum system reserve will increase the total operation costs. The total operation cost incorporates: the DG units' energy production costs, the external suppliers' energy cost, DR program costs, the ESSs and EVs' discharging costs, the non-supplied demand costs, and the generation curtailment power costs [19], [20]. $\Delta t$ represents the duration of the scheduling intervals. This model is adaptable for different scheduling intervals. For instance, for an energy resource scheduling based on a 30-minute scheduling interval, the value of $\Delta \mathrm{t}$ should be 0.5 if the costs of the resources are specified in an hour basis.

$$
\begin{aligned}
& \text { Minimize } O C_{\text {Total }}^{D+1}=
\end{aligned}
$$

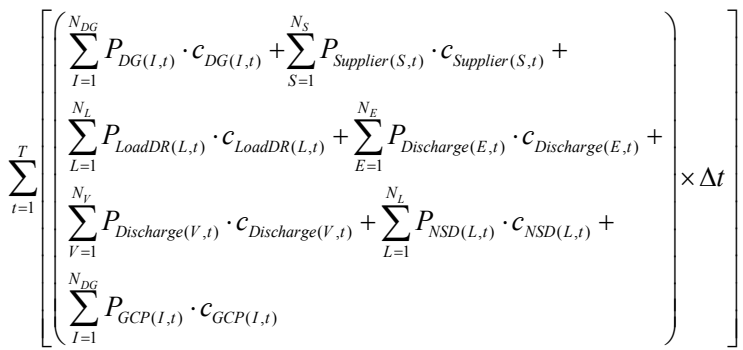

$$
\text { Maximize } M R^{D+1}
$$

The indices are represented by: $E$ is an index of ESSs; $I$ is an index of DG units; $L$ is an index of loads; $S$ is an index of external suppliers; $t$ is an index of time periods; $V$ is an index of EVs;

The parameters are described by: $N_{D G}$ is the number of DG units; $N_{S}$ is the number of external electricity suppliers; $N_{E}$ is the number of ESSs; $N_{L}$ is the number of loads; $N_{V}$ is the number of EVs; $C_{D G(I, t)}$ is the generation price of DG unit $I$ in period $t$ (m.u.); $C_{\text {Supplier(S,t) }}$ is the energy price of external supplier $S$ in period $t$ (m.u.); $C_{\operatorname{LoadDR}(L, t)}$ is the load reduction (DR) cost of load $L$ in period $t$ (m.u.); $C_{\text {Discharge(E,t) }}$ is the discharging cost of ESS $E$ in period $t$ (m.u.); $C_{\text {Discharge(V,t) }}$ is the discharging cost of EV $V$ in period $t$ (m.u.); $C_{N S D(L, t)}$ is the non- 
supplied demand (NSD) cost of load $L$ in period $t$ (m.u.); $C_{G C P(I, t)}$ is the curtailment cost of DG unit $I$ in period $t$ (m.u.).

The variables are described by: $O C$ is the total operation cost (m.u.); $M R^{D+1}$ is the minimum reserve for day ahead (MW) $P_{D G(I, t)}$ is the active power generation of DG unit $I$ in period $t(\mathrm{MW}) ; P_{\text {Supplier }(S, t,)}$ is the active power generation of the external supplier $S$ in period $t(\mathrm{MW}) ; P_{\operatorname{LoadDR}(L, t)}$ is the active power reduction of load $L$ in period $t(\mathrm{MW}) ; P_{\text {Discharge }(E, t,)}$ is the active power discharge of ESS $E$ in period $t(\mathrm{MW}) ; P_{\text {Discharge }(V, t)}$ is the active power discharge of EV $V$ in period $t(\mathrm{MW})$; $P_{\text {Load }(L, t)}$ is the active power demand of load $L$ in period $t(\mathrm{MW})$; $P_{N S D(L, t)}$ is the active power of Non-supplied demand for load $L$ in period $t(\mathrm{MW}) ; P_{G C P(I, t)}$ is the generation curtailment power of DG unit $I$ in period $t$ (MW);

\section{Constraints}

The multi-objective optimization problem with objective functions (1) and (2) is subject to several constraints such as dispatchable DG technical limits, ESS and EVs charging and discharging limits.

The system's net reserve during each period cannot be lower than the minimum reserve. EVs are not considered in the evaluation of the system's net reserve in this case but ESS are considered. The minimum value of the energy stored in the battery and the maximum discharge rate of the battery are evaluated within (3)

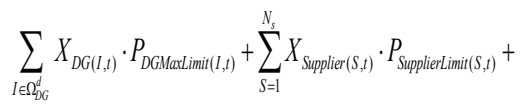

$$
\begin{aligned}
& \sum_{E=1}^{N_{E}} \min \left(E_{\text {Stored }(E, t)}, P_{D \text { ischargelimit }(E, t)}\right) \cdot Y_{(E, t)}+\sum_{I \in \Omega_{D G}^{d i d}} P_{D G(I, t)}-\sum_{L=1}^{N_{L}} P_{\text {Lod }(L, t)} \geq M R^{D+1} \quad \forall t
\end{aligned}
$$

The set in (3) is described by $\Omega_{D G}^{d}$ is a set of dispatchable DG units.

The parameters in (3) are described by: $P_{D G M a x i m i t(I, t)}$ is the maximum active power generation of DG unit $I$ in period $t$ (MW); $P_{\text {Supplierlinit(s,t) }}$ is the maximum active power of upstream supplier $S$ in period $t(\mathrm{MW}) ; P_{\text {Dischargelimit }(\mathrm{E}, t)}$ is the maximum power discharge of ESS $E$ in period $t(\mathrm{MW}) ; P_{\text {Load }(L, t)}$ is the forecasted active power of load $L$ in period $t$ (MW)

The variables in (3) are described by: $X_{D G(I, t)}$ is the binary decision variable of DG unit $I$ in period $t ; X_{\text {Supplier }(S, t)}$ is the binary decision variable of supplier $S$ in period $t ; E_{\text {Sored }(E, t)}$ is the energy stored in ESS unit $E$ at the end of period $t(\mathrm{MWh}) ; Y_{(E, t)}$ is the binary variable of ESS unit $E$ related to power charge in period $t$; $P_{D G(I, t)}$ is the active power generation of DG unit $I$ in period $t$ (MW). Moreover, the minimum reserve should be higher than a defined value $\left(M R_{\min }\right)$ :

$$
M R^{D+1} \geq M R_{\min }
$$

\section{E. Optimization method}

A deterministic Mixed Integer Linear Programming (MILP) methodology was used to solve the scheduling problem. To control the network power flow, a DC model for power balance
(Kirchhoff's first law) is used in this formulation. The model is implemented in TOMLAB and the CPLEX solver is used to solve the problem [21]. A total set of weights are processed corresponding to 100 optimization problems. When the optimizations are solved, a Pareto front algorithm is applied to a set including all the obtained final solutions to find the nondominated solutions (NDS) and discard the dominated solutions. The basic idea of the Pareto front is to pick up the set of points that are Pareto efficient, i.e., the points that are not dominated by other feasible solutions, meaning that they are the best solutions for the multi-objective problem. This set of points constitutes the Pareto optimal set [22], [23].

\section{CASE STUDY}

The method proposed in this paper is tested in a distribution network adapted from [16]. This system is a realistic $30 \mathrm{kV}$ system in Portugal with one substation, 180 buses and 90 load points. The network single-line diagram can be seen in Figure 1. A day-ahead ERM scenario with 24 periods (hours) was developed considering 116 DG units, Demand Response (DR) programs, 7 ESSs and 1000 EVs. It was assumed that the VPP manages the operation of the entire network and the objective is to minimize the total operation costs of the system and to maximize the minimum reserve. The VPP is also responsible for supplying power to the critical loads in the network where DR is not available. We select buses 1-94 for the critical loads. However, loads in bus 95-180 offer the DR programs (in the DR scenario only). A fleet of $1000 \mathrm{EVs}$ with V2G capabilities is considered and the scenario is developed using the tool presented in [24]. The forecasted amount related to EVs trips is 4.50 MWh and a total of 1354 trips.

Table I presents the characteristics of the tested system, namely the considered costs and the capacity of the DG, ESS, $\mathrm{DR}$ and V2G. The PV price is considered as $0.15 \mathrm{~m} . \mathrm{u} . / \mathrm{kWh}$ while its aggregated generation forecast varies between 0 (no sunlight) and 1.49 MW (peak). The wind generation price is set to $0.09 \mathrm{~m} . \mathrm{u} . / \mathrm{kWh}$ and its generation forecast varies between 1.07 and 1.76 MW. The discharging costs considered for the EVs and ESS are set to 0.07 m.u. $/ \mathrm{kWh}$ and $0.06 \mathrm{~m} . \mathrm{u} . / \mathrm{kWh}$, respectively. A charging and discharging efficiency of $90 \%$ is considered for EVs and ESS. The external supplier contract is set to a minimum of $2 \mathrm{MW}$ and a maximum of $10 \mathrm{MW}$ while the price varies along the day. The considered prices and the capacity have taken into account the observations about renewables penetration in [25]. The work is developed in MATLAB R2014a 64 bits, and TOMLAB 64 bits [21], [26].

TABLE I. CHARACTERISTICS OF THE SYSTEM

\begin{tabular}{l|l|l|l}
\hline \hline Resources & $\begin{array}{l}\text { Price } \\
(\mathbf{m . u .} / \mathbf{k W h})\end{array}$ & $\begin{array}{l}\text { Capacity } \\
\text { (MW) }\end{array}$ & $\begin{array}{l}\text { Units } \\
\#\end{array}$ \\
\hline PV & 0.15 & $0.00-1.49$ & 44 \\
\hline Wind & 0.09 & $1.07-1.76$ & 55 \\
\hline Biomass & 0.13 & 1.98 & 17 \\
\hline $\begin{array}{l}\text { External } \\
\text { supplier }\end{array}$ & $0.10-0.16$ & 10.00 & 1 \\
\hline V2G & 0.07 & 3.00 & 1000 \\
\hline ESS & 0.06 & 1.20 & 7 \\
\hline DR & 0.14 & $1.07-1.79$ & 45 \\
\hline \hline
\end{tabular}




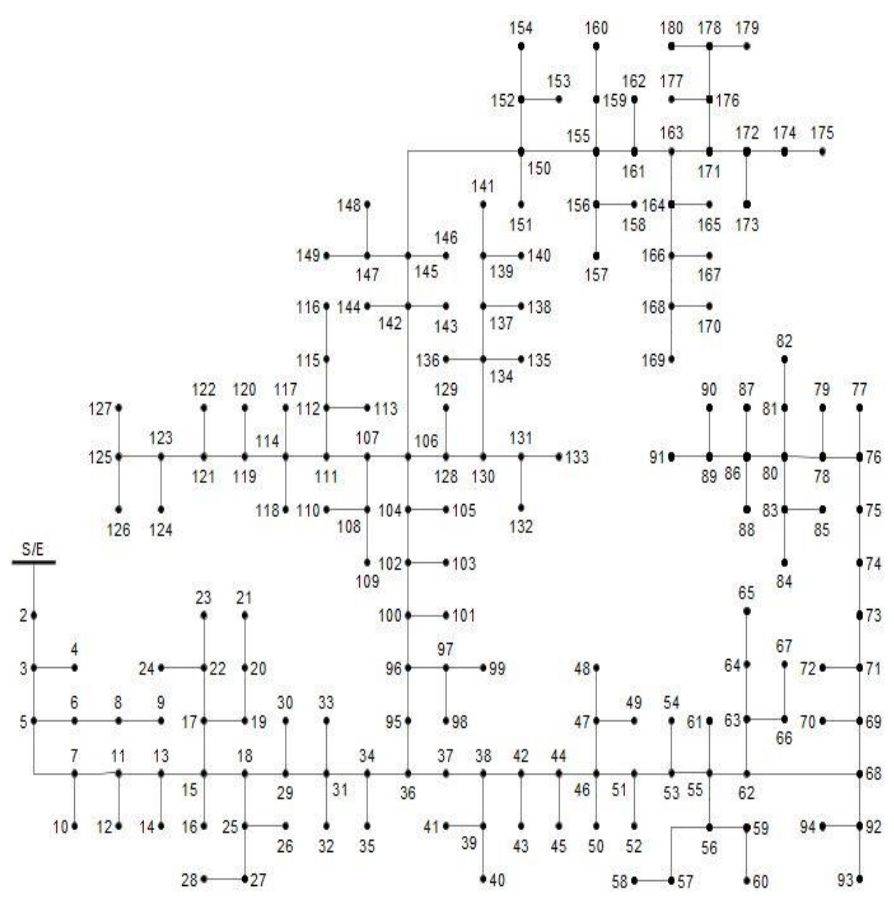

Figure 1. 180-bus 30kV Portuguese network (adapted from [16]).

\section{RESULTS AND DISCUSSION}

The MILP is used in this section to solve the multi-objective scenario. A set of 100 weights are randomly generated for the multi-objective function according to Pareto weighted method and each set is evaluated in the cluster computing platform. After the process regarding the corresponding 100 optimizations, a Pareto front algorithm is run to obtain the nondominated solutions (NDS). Figure 2 depicts the nondominated Pareto fronts for two different scenarios, one scenario considers DR and another not considers, this for the tested approache, namely MILP using TOMLAB. The total number of NDS obtained in MILP with DR was 28, while the total number of NDS without DR was 26 . We selected the solutions to present in the following analysis from the Pareto fronts: lowest total operation cost (NSD-L) and higher total operation cost (NDS-R) (see Figure 2).

Table II shows the 2 selected NDS from the Pareto curves for the two scenarios. In the DR scenario for the NDS-R, the results were $6.93 \mathrm{MW}$ of minimum reserve and 33,005 m.u. of operation costs. In the scenario without DR for the NDS-R, the results were 5.49 MW of minimum reserve and 32,938 m.u. of operation costs. Regarding the NDS-L solution, for the DR scenario, MILP found a solution with 4.26 MW minimum reserve and operation costs, of 31,548 m.u. In terms of the scenario without DR, MILP found a solution with $3.52 \mathrm{MW}$ of minimum reserve and operation costs, of 31,757 m.u. It is possible to see that when considering the use of DR, the solutions found by the MILP approach were significantly better in terms of minimum available reserve.

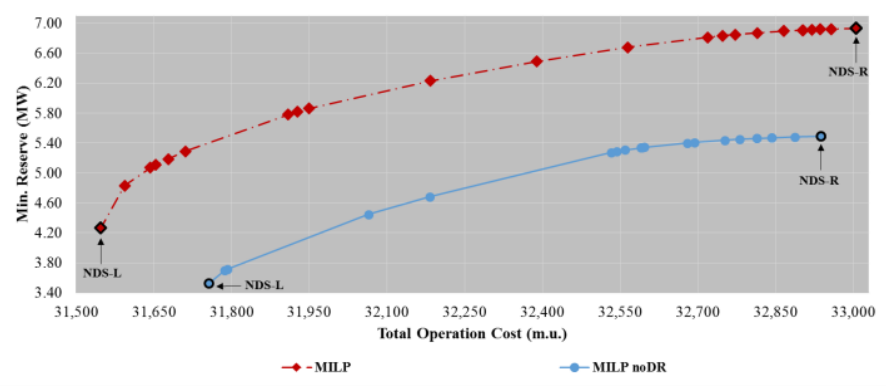

Figure 2. Pareto front obtained in DR scenario (red) and without DR (blue)

TABLE II. SELECTED NON-DOMINATED SOLUTION FROM THE PARETO FRONT

\begin{tabular}{l|l|l|l|l}
\cline { 2 - 5 } & \multicolumn{2}{|l|}{ DR Scenario } & \multicolumn{2}{l}{ noDR Scenario } \\
\cline { 2 - 5 } & NDS-L & NDS-R & NDS-L & NDS-R \\
\hline \hline $\begin{array}{l}\text { Total operation cost } \\
(\mathbf{m . u )}\end{array}$ & 31,548 & 33,005 & 31,757 & 32,938 \\
\hline $\begin{array}{l}\text { Minimum reserve } \\
(\mathrm{MW})\end{array}$ & 4.26 & 6.93 & 3.52 & 5.49 \\
\hline \hline
\end{tabular}

Figures 3 and 4 depicts the optimal energy scheduling results for NDS-L and NDS-R using MILP method, for the DR scenario, respectively. It can be seen that NDS-L used more DR than NDS-R, i.e. a difference of 4.02 MWh. Regarding NSD$\mathrm{R}, \mathrm{ESS}$ and EVs resources were highly charged during the night whereas in NDS-L this was much less, which can be seen in Figure 5 and Figure 6 . The stored energy in ESS allowed the VPP to maintain a higher security of supply, therefore a higher minimum reserve than in NDS-L.

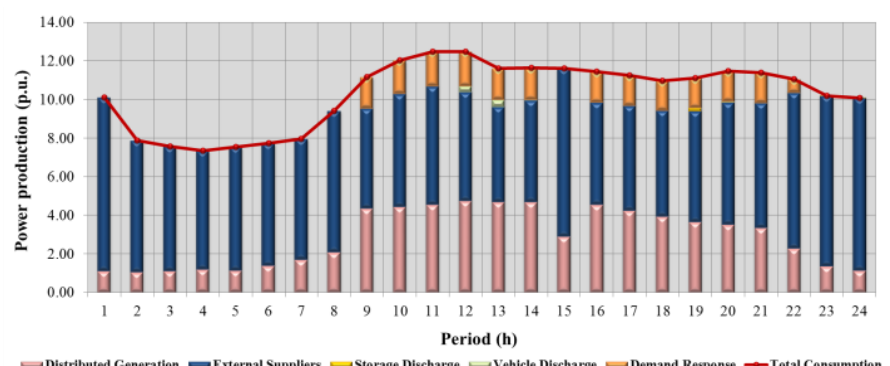

Figure 3. Optimal resource scheduling in MILP for NDS-R.

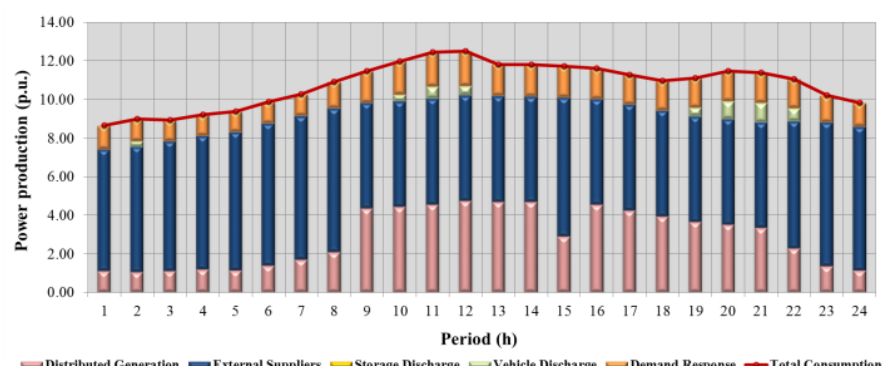

Figure 4. Optimal resource scheduling in MILP for NDS-L. 


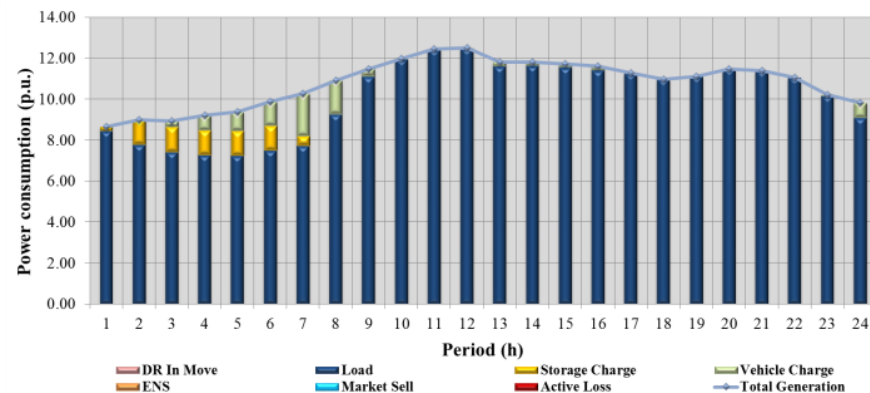

Figure 5. Consumption scheduling in MILP for NDS-R.

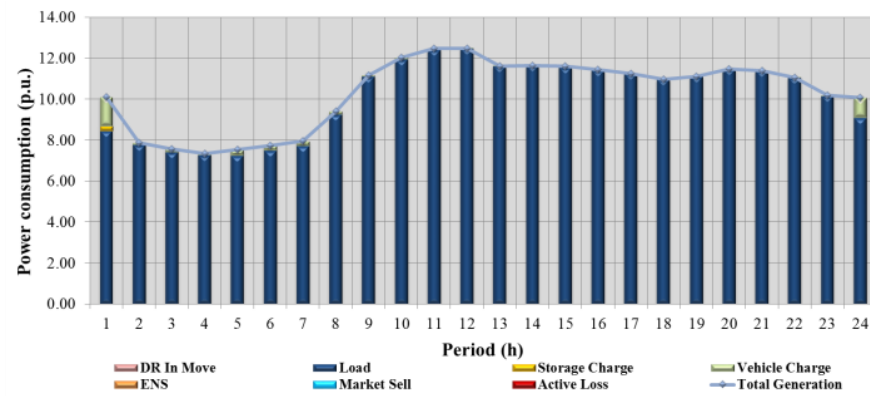

Figure 6. Consumption scheduling in MILP for NDS-L.

\section{CONCLUSIONS}

A decision-making framework to assist the VPPs in the presence of critical loads has been presented. The day-ahead scheduling is designed as a multi-objective problem. The VPPs schedule the resources to obtain a trade-off decision, in order to choose from a reduced operation cost and a safer operation with higher minimum available reseve, which is important with high penetration of critical electricity loads

A deterministic technique based on Mixed-Integer Linear Programming (MILP) is used to solve the multi-objective problem. Two different scenarios were presented, one with DR and another without DR, to assess the influence that the use of DR has in the final results.

In fact, the effect of DR is positive in the minimum available reserve, which was confirmed by our MILP-based methodology.

\section{REFERENCES}

[1] NIST, "Smart Grid: A Beginner's Guide." National Institute of Standards and Technology (NIST), p. 7, 2011.

[2] S. Blumsack and A. Fernandez, "Ready or not, here comes the smart grid!", Energy, vol. 37, no. 1, pp. 61-68, 2012.

[3] M. Wissner, "The Smart Grid - A saucerful of secrets?," Appl. Energy, vol. 88, no. 7, pp. 2509-2518, 2011.

[4] K. Alanne and A. Saari, "Distributed energy generation and sustainable development," Renew. Sustain. Energy Rev., vol. 10, no. 6, pp. 539-558, 2006.

[5] T. Sousa, H. Morais, J. Soares, and Z. Vale, "Day-ahead resource scheduling in smart grids considering Vehicle-to-Grid and network constraints," Appl. Energy, vol. 96, pp. 183-193, 2012.
[6] E. A. Committee, "Keeping the Lights On in a New World," 2009

[7] T. Rahwan and N. Jennings, "Coalition Structure Generation: Dynamic Programming Meets Anytime Optimisation," 2008.

[8] P. Palensky and D. Dietrich, "Demand Side Management: Demand Response, Intelligent Energy Systems, and Smart Loads," IEEE Trans. Ind. Informatics, vol. 7, no. 3, pp. 381-388, Aug. 2011.

[9] C. D. Korkas, S. Baldi, I. Michailidis, and E. B. Kosmatopoulos, "Intelligent energy and thermal comfort management in grid-connected microgrids with heterogeneous occupancy schedule," Appl. Energy, vol. 149, pp. 194-203, 2015.

[10] M. Motevasel, A. R. Seifi, and T. Niknam, "Multi-objective energy management of CHP (combined heat and power)-based micro-grid," Energy, vol. 51, pp. 123-136, 2013.

[11] J. Aghaei and M.-I. Alizadeh, "Multi-objective self-scheduling of CHP (combined heat and power)-based microgrids considering demand response programs and ESSs (energy storage systems)," Energy, vol. 55, pp. 1044-1054, Jun. 2013.

[12] A. Chaouachi, R. M. Kamel, R. Andoulsi, and K. Nagasaka, "Multiobjective Intelligent Energy Management for a Microgrid," IEEE Trans. Ind. Electron., vol. 60, no. 4, pp. 1688-1699, Apr. 2013.

[13] G. R. Aghajani, H. A. Shayanfar, and H. Shayeghi, "Presenting a multiobjective generation scheduling model for pricing demand response rate in micro-grid energy management," Energy Convers. Manag., vol. 106, pp. 308-321, Dec. 2015.

[14] M. Motevasel and A. R. Seifi, "Expert energy management of a microgrid considering wind energy uncertainty," Energy Convers. Manag., vol. 83, pp. 58-72, 2014.

[15] T. Niknam, R. Azizipanah-Abarghooee, and M. R. Narimani, "An efficient scenario-based stochastic programming framework for multiobjective optimal micro-grid operation," Appl. Energy, vol. 99, pp. 455470, Nov. 2012

[16] J. Soares, H. Morais, and Z. Vale, "Particle Swarm Optimization based approaches to vehicle-to-grid scheduling," in 2012 IEEE Power and Energy Society General Meeting, 2012, pp. 1-8.

[17] T. Crnko, "Life Safety Loads Depend On Reliable Power Systems," IAEI Magazine, vol. September-. IAEI, 2012.

[18] W. Su, J. Wang, and J. Roh, "Stochastic Energy Scheduling in Microgrids With Intermittent Renewable Energy Resources," IEEE Trans. Smart Grid, vol. 5, no. 4, pp. 1876-1883, Jul. 2014.

[19] T. Sousa, T. Pinto, H. Morais, Z. Vale, and Ieee, "Intelligent Electric Vehicle Heuristic for Energy Resource Management using Simulated Annealing," 2012 3rd IEEE PES Innovative Smart Grid Technologies Europe. 2012.

[20] J. Soares, T. Sousa, H. Morais, Z. Vale, and P. Faria, "An optimal scheduling problem in distribution networks considering V2G," 2011 IEEE Symposium on Computational Intelligence Applications In Smart Grid CIASG. IEEE, pp. 1-8, 2011.

[21] TOMLAB, "TOMLAB optimization," 2015. [Online]. Available: http://tomopt.com/tomlab/about/.

[22] J. L. Bernal-Agustín and R. Dufo-López, "Multi-objective design and control of hybrid systems minimizing costs and unmet load," Electr. Power Syst. Res., vol. 79, no. 1, pp. 170-180, Jan. 2009.

[23] R. Dufo-López, J. L. Bernal-Agustín, J. M. Yusta-Loyo, J. A Domínguez-Navarro, I. J. Ramírez-Rosado, J. Lujano, and I. Aso, "Multi-objective optimization minimizing cost and life cycle emissions of stand-alone PV-wind-diesel systems with batteries storage," Appl. Energy, vol. 88, no. 11, pp. 4033-4041, Nov. 2011.

[24] J. Soares, B. Canizes, C. Lobo, Z. Vale, and H. Morais, "Electric Vehicle Scenario Simulator Tool for Smart Grid Operators," Energies, vol. 5, no. 12, pp. 1881-1899, Jun. 2012.

[25] A. Zervos, C. Lins, and J. Muth, RE-thinking 2050: a 100\% renewable energy vision for the European Union. EREC, 2010.

[26] MathWorks, "MATLAB - The Language Of Technical Computing." [Online]. Available: http://www.mathworks.com/products/matlab/. 